\title{
Re: Risk of ESRD in Prior Living Kidney Donors
}

\author{
Wainright $\mathrm{JL}^{1}$, Robinson $\mathrm{AM}^{1}$, Wilk $\mathrm{AR}^{1}$, Klassen $\mathrm{DK}^{2}$, Cherikh WS${ }^{1}$, Stewart $\mathrm{DE}^{1}$ \\ ${ }^{1}$ Research Department, United Network for Organ Sharing, Richmond, USA \\ ${ }^{2}$ Office of the Chief Medical Officer, United Network for Organ Sharing, Richmond, USA
}

Am J Transplant 2018;18:1129-1139. doi: 10.1111/ajt.14678.

\section{EDITORIAL COMMENT}

Every year more living kidney donors (LKDs) are requiring renal replacement therapies due to end-stage renal disease (ESRD). In this retrospective observational study, the authors have evaluated the association between characteristics at donation and development of ESRD for LKDs who have donated between 1994 and $2016(n=123.526)$ in the United States by using Organ Procurement and Transplantation Network and Centers for Medicare and Medicaid Services data. Of those, 218 LKDs developed ESRD, with a median of 11.1 years between donation and ESRD. Although the overall absolute 20-year risk of ESRD was low for LKDs, it was not uniform either. In multivariable analysis, being male, black race, having lower estimated glomerular filtration rate, having higher body mass index at donation, being first-degree relative of the recipient and full sibling were identified as significant risk factors for ESRD. There was also a strong age and race interaction, with older age at donation conferring higher risk for white donors, but younger age at donation resulting higher risk for black donors. Interestingly LKDs from low socioeconomic levels had a higher risk of ESRD compared to those living in higher income neighborhoods. It is important for the potential living donors to understand their absolute risk at the time of decision making for donation. Also, it is crucial to build national donor follow-up programs for calculating the absolute and relative risks for donors.

Yarkın Kamil Yakupoğlu, MD

बCopyright 2018 by the Association of Urological Surgery / Journal of Urological Surgery published by Galenos Publishing House. 